\title{
OPTIMIZACIÓN DE LA PRODUCCIÓN DE LA ENZIMA L- ARABINOSA ISOMERASA POREnterococcus faecium DBFIQ E36 MEDIANTE TÉCNICAS ESTADÍSTICAS MULTIVARIADAS
}

\author{
R. M. Manzo ${ }^{1}$, A. C. Simonetta $^{2}$, L. R. B. Gonçalves ${ }^{3}$ y E. J. Mammarella ${ }^{1}$ \\ ${ }^{1}$ Instituto de Desarrollo Tecnológico para la Industria Química (INTEC)-CONICET- \\ Universidad Nacional del Litoral (UNL), Santa Fe, Argentina \\ ${ }^{2}$ Cát. deMicrobiología y Biotecnología, Departamento de Ing. de Alimentos, Facultad de \\ Ingeniería Química, Universidad Nacional del Litoral, Santa Fe, Argentina \\ ${ }^{3}$ DEQ - Universidade Federal do Ceará, Fortaleza, Ceará, Brasil \\ E-mail para contato: ejoma@intec.unl.edu.ar
}

\begin{abstract}
RESUMEN - El estudio de los azúcares raros se ha visto incrementado dados los avances en relación con el entendimiento de su estructura-función y de las potenciales aplicaciones que poseen. La D-tagatosa ha surgido como uno de los nutraceúticos más promisorios por sus numerosas propiedades funcionales. Simultáneamente, se ha demostrado que es posible obtener dicho azúcar mediante el empleo de la enzima L-arabinosa isomerasa (EC 5.3.1.4). Para incrementar las concentraciones de la enzima obtenida de extractos libres de células provenientes de la cepa Enterococcus faecium DBFIQ E36, fue optimizada la composición del medio de cultivo. El medio de cultivo MRS modificado permitió obtener extractos libres de células con concentraciones de proteínas de $15,2 \mathrm{mg} \mathrm{ml}^{-1}$, representando un aumento de 58 veces en relación al medio no optimizado.
\end{abstract}

\section{INTRODUCCIÓN}

La obtención de D-tagatosa a partir de D-galactosa por medios biológicos utilizando la enzima L-arabinosa (D-galactosa) isomerasa (EC 5.3.1.4), es un proceso de alto interés tecnológico, particularmente en la industria de productos alimenticios, como endulzante de alimentos especiales (Boudebbouze et al., 2011). El interés en este cetoazúcar reside en que la D-tagatosa es un azúcar raro que posee numerosos efectos beneficiosos para la salud por tratarse de un endulzante hipocalórico que promueve la pérdida de peso (Moore, 2006), que posee un efecto glucémico nulo y efecto prebiótico reconocido, además de no poseer efectos colaterales indeseados.De esta forma, a partir de la cepa Enterococcus faecium DBFIQ E36, previamente caracterizada como productora de la enzima L-arabinosa isomerasa, se efectuaron ensayos microbiológicos de optimización de la producción de dicha enzima.

Definiendo calidad como la consistencia del desempeño alrededor de un objetivo (valor nominal o promedio, en caso de que se disponga), minimizando simultáneamente la desviación estándar (Roy, 2001), el diseño de Taguchi fue empleado para analizar la influencia de los diferentes componentes del medio comercial MRS, de manera de incrementar la producción de nuestra enzima minimizando simultáneamente los costos de fabricación de los medios, lo que llega a representar hasta el 50\% del costo total de un proceso de fermentación con microorganismos. De esta forma, aquellas otras variables 
operativas que pueden influir en la producción final de la enzima, o fueron mantenidos constantes u omitidos.Así, el acercamiento o propuesta de Taguchi, es un tipode diseño experimental desarrollado para incrementar la calidad de los productos yahorrar dinero en las industrias, por ello, para la aplicación del mencionado diseño, sehan creado un número de arreglos ortogonales especiales, cada uno de los cuales esutilizado para un cierto número de experiencias. Dichos arreglos pretenden obtener, enel menor número de experiencias posibles, la representación y significancia de todas lasvariables incluidas en el análisis. Luego, para analizar los resultados, se emplea larelación señal-ruido para obtener un diseño robusto a la influencia de factoresincontrolables.

Así, el objetivo de este trabajo ha sido diseñar y optimizar medios de cultivo de manera de maximizar la producción dela enzima buscadaempleando el método de Taguchi como herramienta estadística de diseño y análisis de los resultados.

\section{MATERIALES Y MÉTODOS}

\subsection{Ensayos de modificación de la composición del medio de cultivo para aumentar la producción de la enzima L-arabinosa isomerasa}

Para la realización de dicho estudio de optimización (Tabla 1), se estudiaron 11 variables diferentes, resultando ser cada una de ellas, los componentes del medio de cultivo ManRogosa-Sharpe (MRS), que han sido testeados en tres niveles diferentes, es decir, a tres concentraciones diferentes (Givry y Duchiron, 2008). Finalmente, se realizaron 27 experiencias independientes evaluando 11 efectos a 3 concentraciones diferentes, teniendo en cuenta el número de pruebas mínimas dadas por el diseño de arreglos ortogonales seleccionado, el cual fue L-27 $\left(3^{* 11}\right)$. Para el análisis de los datos estadísticos, se empleó el software Minitab versión 15.0 (Minitab Inc., Pennsylvania, EEUU).

Para la realización de las experiencias, se partió de la cepa E. faecium DBFIQ E36 previamente conservada a $-80^{\circ} \mathrm{C}$ y se efectuó la producción de los extractos de acuerdo a Manzo et al. (2013). Los extractos estériles fueron almacenados y posteriormente, se les determinó por triplicado, la actividad enzimática y la concentración de proteínas. Asimismo, cada experiencia fue realizada por duplicado, en forma independiente y al azar (Im et al., 2009). Finalmente, se utilizó como cultivo control del crecimiento y de la producción de la enzima, el crecimiento de la cepa seleccionada en medio MRS comercial.

Tabla 1 - Tratamientos y niveles seleccionados para el desarrollo del diseño de Taguchi para optimizar la producción enzimática.

\begin{tabular}{ccccc}
\hline \multirow{2}{*}{ Factor } & Componentes $\left(\mathbf{g ~ l}^{\mathbf{- 1}}\right)$ & $\mathbf{3}$ & $\mathbf{2}$ & Niveles \\
\cline { 3 - 5 } & & $\mathbf{1}$ & 10 & 20 \\
\hline 1 & Peptona & 5 & 10 & 20 \\
2 & Extracto de carne & 5 & 5 & 10 \\
3 & Extracto de levadura & 2,5 & 2 & 4 \\
4 & Fosfato Dipotásico & 1 & 5 & 10 \\
5 & Acetato de Sodio & 2,5 & 2 & 4 \\
6 & Citrato de amonio & 1 & 5 & 10 \\
7 & D-glucosa & 1 & 1,08 & 2,16 \\
8 & Tween 80* & 0,5 & 0,4 & 0,8 \\
9 & Sulfato de Magnesio & 0,2 & &
\end{tabular}




\begin{tabular}{ccccc}
\hline 10 & Sulfato de Manganeso & 0,2 & 0,4 & 0,8 \\
11 & L-arabinosa & 5 & 10 & 20 \\
\hline
\end{tabular}

*La densidad del Tween 80 es $1,08 \mathrm{~g} \mathrm{ml}^{-1}$.

\subsection{Ensayos de determinación de proteínas y de actividad enzimática}

El ensayo enzimático fue realizado de acuerdo a la metodología propuesta por Torres et al. (2014), mientras que la D-tagatosa producida fue detectada empleando el ensayo de Kulka (1956). La concentración de proteína de los diferentes extractos obtenidos fue medida empleando el ensayo de Bradford (1976).

\section{RESULTADOS Y DISCUSIÓN}

\subsection{Modificación de la composición del medio de cultivo MRS para optimizar la producción de la enzima $L$-arabinosa isomerasa}

Para este estudio, la respuesta analizada ha sido la actividad enzimática específica de la enzima L-arabinosa isomerasa por gramo de células obtenida luego del crecimiento de la cepa seleccionada E. faecium DBFIQ E36 en los diferentes medios de cultivo MRS modificados oportunamente para el desarrollo de cada experiencia.En la Tabla 2, se aprecian los resultados obtenidos luego de la realización de las diversas pruebas de crecimiento en los medios elaborados, producción de los extractos enzimáticos y medición de la actividad enzimática de todos ellos. Cada prueba fue realizada por triplicado presentándose los valores promedio.

Tabla 2-Optimización de la actividad enzimática empleando arreglos ortogonales de Taguchi de 27 experiencias a tres niveles y 11 factores.

\begin{tabular}{cccccccc}
\hline Exp. & {$[\mathbf{P}](\mathbf{m g}$} & \multicolumn{2}{c}{$\mathbf{A E}\left(\mathbf{U ~ m}^{\mathbf{1}}\right)^{*}$} & \multicolumn{2}{c}{ AE esp. $\left(\mathbf{U ~} \mathbf{~ m g}^{-\mathbf{1}}\right)^{*}$} & \multicolumn{2}{c}{ AE esp. $\left(\mathbf{U ~}^{\mathbf{- 1}} \mathbf{~ m}^{\mathbf{- 1}}\right)^{*}$} \\
\cline { 3 - 8 } $\mathbf{N}^{\circ}$ & $\left.\mathbf{~ m l}^{\mathbf{1}}\right)$ & $\boldsymbol{\mu}$ & $\boldsymbol{\sigma}$ & $\boldsymbol{\mu}$ & $\boldsymbol{\sigma}$ & $\boldsymbol{\mu}$ & $\boldsymbol{\sigma}$ \\
\hline $\mathbf{1}$ & 3,0 & 2,738 & 3,606 & 0,913 & 1,202 & 0,608 & 0,801 \\
$\mathbf{2}$ & 1,0 & 10,809 & 1,570 & 10,809 & 1,570 & 8,752 & 1,271 \\
$\mathbf{3}$ & 15,3 & 171,558 & 2,174 & 11,189 & 0,142 & 4,833 & 0,061 \\
$\mathbf{4}$ & 9,8 & 148,490 & 6,159 & 15,186 & 0,630 & 7,047 & 0,292 \\
$\mathbf{5}$ & 11,6 & 127,597 & 4,106 & 11,042 & 0,355 & 3,693 & 0,119 \\
$\mathbf{6}$ & 0,8 & 5,133 & 2,174 & 6,162 & 2,610 & 3,081 & 1,305 \\
$\mathbf{7}$ & 7,5 & 280,737 & 4,589 & 37,432 & 0,612 & 17,131 & 0,280 \\
$\mathbf{1 8}$ & 10,3 & 110,326 & 3,502 & 10,677 & 0,339 & 4,262 & 0,135 \\
$\mathbf{9}$ & 9,2 & 23,128 & 5,193 & 2,523 & 0,567 & 0,837 & 0,188 \\
$\mathbf{1 0}$ & 12,4 & 81,582 & 3,744 & 6,556 & 0,301 & 3,961 & 0,182 \\
$\mathbf{1 1}$ & 5,5 & 105,616 & 0,483 & 19,203 & 0,088 & 4,263 & 0,019 \\
$\mathbf{1 2}$ & 2,2 & 27,838 & 3,623 & 12,528 & 1,631 & 3,952 & 0,514 \\
$\mathbf{1 3}$ & 10,1 & 281,622 & 26,816 & 27,762 & 2,644 & 7,277 & 0,693 \\
$\mathbf{1 4}$ & 10,1 & 68,539 & 4,469 & 6,801 & 0,443 & 2,657 & 0,173 \\
$\mathbf{1 5}$ & 13,6 & 509,360 & 0,242 & 37,574 & 0,018 & 15,656 & 0,007 \\
$\mathbf{1 6}$ & 15,2 & 425,060 & 5,797 & 27,924 & 0,381 & 13,172 & 0,180 \\
$\mathbf{1 7}$ & 14,8 & 453,804 & 5,556 & 30,708 & 0,376 & 13,770 & 0,169 \\
$\mathbf{1 8}$ & 9,8 & 127,838 & 3,623 & 13,001 & 0,368 & 8,966 & 0,254 \\
$\mathbf{1 9}$ & 9,3 & 332,065 & 6,522 & 35,791 & 0,703 & 9,015 & 0,177 \\
$\mathbf{2 0}$ & 14,2 & 100,664 & 1,087 & 7,078 & 0,076 & 2,445 & 0,026 \\
$\mathbf{2 1}$ & 4,2 & 150,664 & 0,121 & 35,686 & 0,029 & 10,359 & 0,008 \\
$\mathbf{2 2}$ & 13,0 & 394,465 & 59,159 & 30,343 & 4,551 & 11,054 & 1,658 \\
\hline
\end{tabular}




\begin{tabular}{cccccccc}
\hline $\mathbf{2 3}$ & 8,6 & 252,114 & 2,536 & 29,466 & 0,296 & 16,462 & 0,166 \\
$\mathbf{2 4}$ & 11,3 & 0,000 & 0,000 & 0,000 & 0,000 & 0,000 & 0,000 \\
$\mathbf{2 5}$ & 11,6 & 68,539 & 2,536 & 5,909 & 0,219 & 2,373 & 0,088 \\
$\mathbf{2 6}$ & 13,9 & 461,051 & 7,488 & 33,195 & 0,539 & 6,503 & 0,106 \\
$\mathbf{2 7}$ & 9,6 & 224,577 & 7,126 & 23,367 & 0,741 & 8,482 & 0,269 \\
\hline
\end{tabular}

\$ 1 U: $\mu \mathrm{mol} \mathrm{min}^{-1}$ bajo las condiciones ensayadas; *valores de actividad enzimática multiplicados por $1 \times 10^{4} ;+$ Los datos son el promedio de tres réplicas \pm la desviación estándar incluyendo el error experimental; $\mu$ : promedio; $\sigma$ : desviación estándar.

Los medios 3, 16 y 17 fueron los que permitieron obtener una mayor concentración de proteínas, mientras que las mejores actividades enzimáticas se lograron para los medios 15, 16 y 17. Asimismo, todos los extractos libres de células presentaron actividad enzimática. Estos resultados confirmarían la relación directa entre una mayor concentración de proteínas y una mayor actividad enzimática. En la Figura 1puede observarse la influencia de los componentes del medio de cultivo MRS, sobre la producción de la enzima en estudio. Tales resultados están expresados a partir de los efectos medios que tiene cada componente sobre la actividad enzimática y no sobre la variación de dicha actividad al modificar la concentración de cada componente en particular.
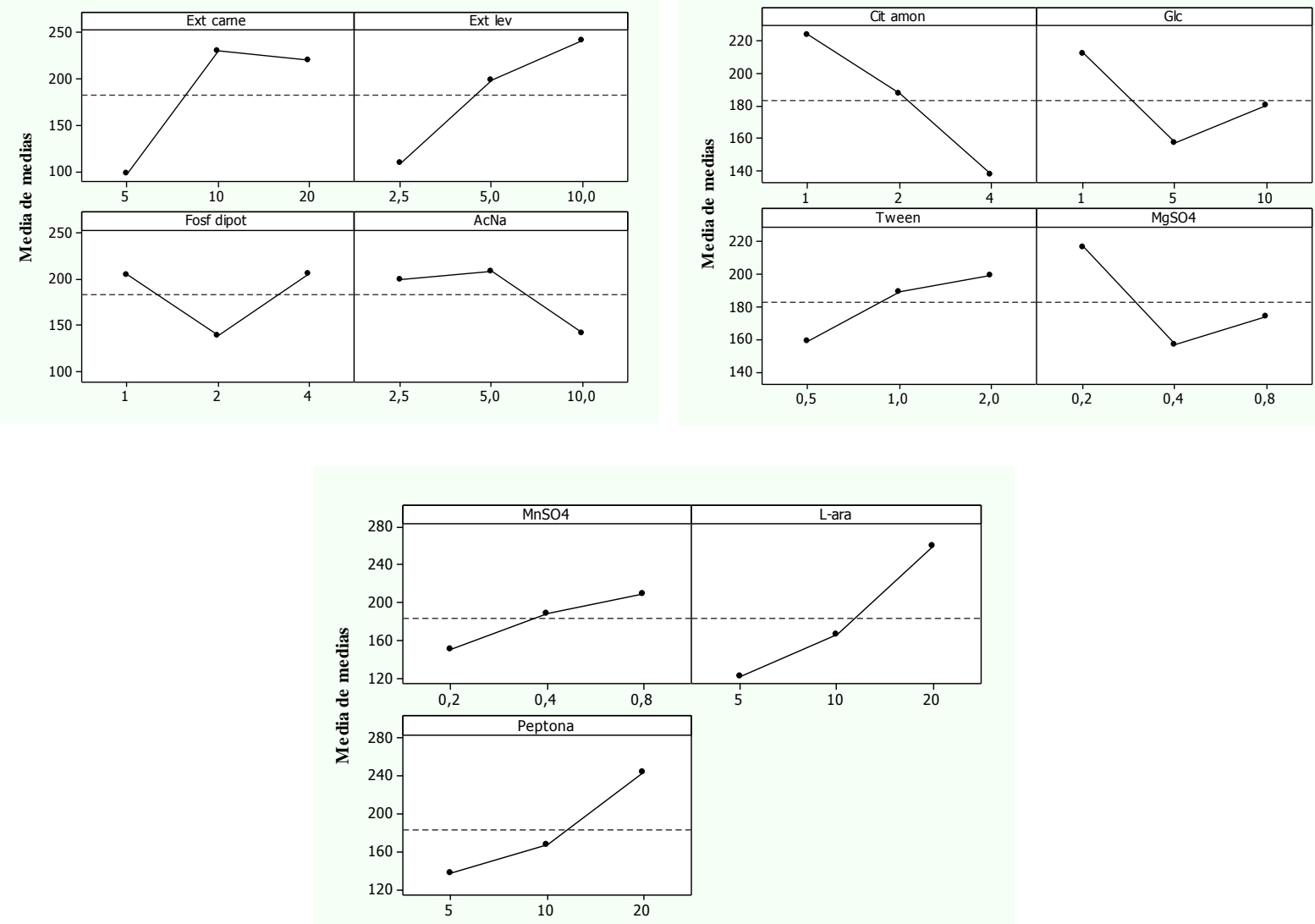

Figura 1- Influencia de los efectos principales sobre la optimización de la respuesta de la actividad enzimática, los cuales muestran la performance promedio de cada factor a un dado nivel.

Observando la Figura 1, puede apreciarse que el aumento en el agregado de D-glucosa, citrato de amonio, $\mathrm{MgSO}_{4}$ y acetato de sodio produjeron un efecto negativo sobre la producción de la 
enzima en los diferentes extractos, mientras que el aumento en las concentraciones de extracto de levadura, Tween $80, \mathrm{MnSO}_{4}$, L-arabinosa y peptona han producido efectos positivos en la respuesta analizada.

El efecto de los diferentes componentes del medio MRS sobre la producción de la enzima Larabinosa isomerasa ha sido escasamente estudiado, siendo Givry y Duchiron (2008), los únicos que han estudiado en forma univariada, la optimización del medio de cultivo MRS para la producción de la enzima L-arabinosa isomerasa producida por Lactobacillus bifermentans DSM $20003^{\mathrm{T}}$. Esta cepa bacteriana pertenece al grupo de bacterias del ácido láctico, siendo también, un organismo mesófilo y GRAS, por lo que sus resultados son comparables con aquellos obtenidos por E. faecium DBFIQ E36.

La influencia de la fuente de carbono principal, L-arabinosa, es esencial para la producción de la enzima L-arabinosa isomerasa. De hecho, la misma es una enzima inducible producida por la bacteria cuando la aldosa L-arabinosa se encuentra presente en el medio de crecimiento (Dobrogosz y De Moss, 1963). Asimismo, el cultivo bacteriano en un medio con baja concentración de D-glucosa y en presencia de una concentración mayor de L-arabinosa, condujo a un crecimiento celular inicial debido al consumo de la D-glucosa seguido por una inducción de las enzimas de la vía de la fosfocetolasa y un crecimiento celular adicional como consecuencia del consumo de la pentosa (Yamanaka y Wood, 1966). En este sentido, lo desarrollado en el párrafo anterior explica el efecto que posee la D-glucosa sobre el cultivo de E. faecium DBFIQ E36, donde una baja concentración de D-glucosa y en presencia de Larabinosa, conlleva a una producción mayor de la enzima pero, el aumento de la concentración de la hexosa, esta última actúa como represora de la expresión de la enzima, disminuyendo así, la cantidad final de enzima obtenida.

Al igual que L. bifermentans, la cepa de E. faecium ha logrado desarrollarse en ausencia de una fuente de nitrógeno orgánico (Deshmukh et al., 1994). En primer lugar, el extracto de levadura ha sido la fuente orgánica de nitrógeno que mayor influencia positiva ejerció sobre la actividad enzimática obtenida, resultados que coinciden con Givry y Duchiron, (2008). En segundo lugar, la variación de la concentración de peptona evidenció un efecto positivo sobre la actividad enzimática, al aumentar su concentración, pero dicho influencia no fue tan intensa como ocurrió con el extracto de levadura. Además, el aumento en la concentración de extracto de carne, arrojó un leve aumento de la actividad, aunque, un incremento aún mayor, hasta 20 $\mathrm{g} \mathrm{l}^{-1}$, ya no produjo ningún incremento, manteniéndose la actividad enzimática constante.

Con respecto al efecto que poseen los iones metálicos sobre la producción de la enzima, el ión $\mathrm{Mn}^{2+}$, conocido cofactor y activador de la enzima L-arabinosa isomerasa, incrementa profundamente la actividad enzimática a medida que su concentración aumenta. En contraposición, el catión $\mathrm{Mg}^{2+}$ ejerce una actividad inhibitoria sobre la enzima en estudio, por lo que la actividad enzimática decae al aumentar su concentración.En síntesis, que la actividad enzimática en relación al incremento del catión $\mathrm{Mn}^{2+}$ aumente, no significa que la bacteria produzca en forma estricta una mayor cantidad de enzima, sino que la combinación de diversos factores, tales como actividad (actividad en función de la concentración del catión), estabilidad (actividad en función del tiempo y condiciones ambientales), crecimiento celular obtenido, son los que han propiciado una mayor actividad enzimática luego de la optimización de la producción. 
Por otro lado, el análisis de la varianza ha sido utilizado para evaluar los resultados del experimento de arreglos ortogonales y para determinar con cuanta variación estadística cada factor o efecto principal, ha contribuido al ensayo en sí (Tabla 3).

Tabla 3 - Análisis de la varianza (ANOVA) de los resultados experimentales sobre la producción de la enzima L-arabinosa isomerasa por E. faecium DBFIQ E36.

\begin{tabular}{|c|c|c|c|c|c|c|c|}
\hline Factores & $v$ & $\begin{array}{c}\text { Suma de } \\
\text { cuadrados } \\
\text { (residuos) }\end{array}$ & $\begin{array}{l}\text { Suma } \\
\text { Pura }\end{array}$ & $\begin{array}{c}\text { Varianza } \\
\left(\mathbf{M S}_{\mathbf{E}}\right)+\end{array}$ & $\begin{array}{l}\text { Valor } \\
\text { de F } \$\end{array}$ & $\begin{array}{l}\text { Valor } \\
\text { de } p^{\dagger}\end{array}$ & $\begin{array}{c}\% \text { de } \\
\text { contribución* }\end{array}$ \\
\hline $\begin{array}{l}\text { Extracto de } \\
\text { carne }\end{array}$ & 2 & 118691 & 191746 & 95873 & 7,82 & $0,065 \dagger$ & 19,35 \\
\hline L-arabinosa & 2 & 99883 & 148418 & 74209 & 6,05 & $0,089 \dagger$ & 16,28 \\
\hline $\begin{array}{l}\text { Extracto de } \\
\text { levadura }\end{array}$ & 2 & 95680 & 150982 & 75491 & 6,15 & $0,087 \dagger$ & 15,59 \\
\hline Peptona & 2 & 81692 & 81692 & 40846 & 3,33 & 0,173 & 13,31 \\
\hline $\begin{array}{c}\text { Sulfato de } \\
\text { Manganeso }\end{array}$ & 2 & 62117 & 97665 & 48832 & 3,98 & 0,143 & 10,12 \\
\hline $\begin{array}{l}\text { Sulfato de } \\
\text { Magnesio }\end{array}$ & 2 & 48901 & 106821 & 53411 & 4,35 & 0,130 & 7,97 \\
\hline $\begin{array}{c}\text { Fosfato } \\
\text { dipotásico }\end{array}$ & 2 & 47303 & 101987 & 50994 & 4,16 & 0,137 & 7,71 \\
\hline $\begin{array}{l}\text { Citrato de } \\
\text { amonio }\end{array}$ & 2 & 12845 & 13277 & 6638 & 0,54 & 0,630 & 2,09 \\
\hline Glucosa & 2 & 4993 & 25200 & 12600 & 1,03 & 0,457 & 0,81 \\
\hline $\begin{array}{l}\text { Acetato de } \\
\text { sodio }\end{array}$ & 2 & 4004 & 9901 & 4950 & 0,40 & 0,699 & 0,65 \\
\hline Tween 80 & 2 & 639 & 23326 & 11663 & 0,95 & 0,479 & 0,10 \\
\hline $\begin{array}{c}\text { Error } \\
\text { Residual }\end{array}$ & 3 & 36799 & 36799 & 12266 & & & 6,00 \\
\hline Total & 25 & 613547 & & & & & 100,00 \\
\hline
\end{tabular}

v: grados de libertad; * El porcentaje de contribución ha sido calculado para cada factor como la relación entre la suma de cuadrados para cada factor y la suma total de los cuadrados; $\$ \mathrm{El}$ valor de la prueba $\mathrm{F}$ se calcula como la relación entre la varianza de cada factor y la varianza del error residual; + En este caso, la varianza es calculada como la relación entre la suma pura y los grados de libertad; $\uparrow$ Valores significativos para un $90 \%$ de confianza. El valor $\mathrm{F}$ para una probabilidad $\alpha=0,1$ y 2 grados de libertad $(v)$ para el numerador y tres $v$ para el denominador es: 5.462 .

La variabilidad de los datos experimentales fue explicada en términos de los efectos significativos. Los cinco factores que más influencia han poseído sobre la producción de la enzima L-arabinosa isomerasa, explicaron el 74,65\% de la contribución del ensayo. Los efectos principales extracto de carne, L-arabinosa y extracto de levadura fueron significativos para un $90 \%$ de confianza. Los componentes peptona, sulfato de magnesio y sulfato de manganeso, aunque no fueron estadísticamente significativos para un $90 \%$ de confianza, los valores de sus pruebas $\mathrm{F}$ estuvieron cerca del valor de $\mathrm{F}$ crítico, por lo que no deben descartarse y deberán ser tenidas en cuenta para un posterior análisis de optimización. 
En general, el diseño de Taguchi es una metodología que se enfoca sobre los efectos principales (factores) de los diseños. Sin embargo, permite analizar interacciones entre dos factores que sean esenciales para el análisis global del proceso. De esta forma, que existan un mayor número de factores en un estudio, aumenta la probabilidad de que exista un cierto número de interacciones con una influencia importante sobre el sistema. En estos casos, la herramienta índice de severidad de interacción para dos factores, permite obtener valores de una interacción determinada, de manera de mostrar la influencia de dos factores individuales a varios niveles de interacción. Esto indicaría la potencial influencia de las interacciones sobre el modelo experimental, en caso de que las mismas hayan sido incluidas en el diseño propiamente dicho (resultados no mostrados). Se ha demostrado la presencia de interacciones muy fuertes entre ciertos componentes del medio. Simultáneamente, el error residual del ANOVA ha sido considerable, tal como se observa en la Tabla 3. Esto se debe principalmente a que, en el diseño propiamente dicho, ninguna interacción fue considerada, sino que sólo los efectos principales fueron analizados. Asimismo, la particularidad que poseen estos ensayos de arreglos ortogonales es que debe tenerse un conocimiento a priori de las interacciones presentes, en caso de que existan, de manera de poder incluirlas en el análisis de la varianza, siendo de hecho la principal limitación de este ensayo, dado que no se poseía un conocimiento previo de la influencia de cada componente sobre la producción de la enzima. Como alternativa, el método tiene la posibilidad de calcular un índice de interacción entre factores, a nivel gráfico o a través del índice de severidad, aunque calcula la mencionada interacción no en forma individual, sino como la diferencia entre los dos efectos principales correspondientes a la interacción.

\section{CONCLUSIONES}

La realización de este estudio ha sido muy provechoso, dado que ha permitido incrementar los niveles de la enzima L-arabinosa isomerasa y conocer, en forma preliminar, el comportamiento de los diferentes componentes del medio teniendo en cuenta futuros estudios de optimización a efectuarse en biorreactores en condiciones de $\mathrm{pH}$, temperatura, oxígeno disuelto y condiciones reductoras controladas. Asimismo, debido a los pocos estudios disponibles en relación a la optimización de la producción de la enzima, ha sido imposible poder predecir las posibles interacciones que podrían haber influido en el ensayo, de manera de efectuar una adecuado diseño de variables principales e interacciones, de modo de minimizar el error residual y de obtener un estudio representativo de la optimización de la producción de la enzima L-arabinosa isomerasa.

La composición optimizada del medio de cultivo MRS para la producción de la enzima L-arabinosa isomerasa por E. faecium DBFIQ E36, se indica a continuación donde la concentración de cada componente está expresada en $\mathrm{g}^{-1}$ : peptona: 20 , extracto de carne: 10 , extracto de levadura: 10, Fosfato dipotásico $\left(\mathrm{K}_{2} \mathrm{HPO}_{4}\right)$ : 1, Acetato de sodio (AcNa): 5, Citrato de amonio: 1, Glucosa: 1, Tween 80: 2, Sulfato de Magnesio $\left(\mathrm{MgSO}_{4}\right)$ : 0.2, Sulfato de Manganeso $\left(\mathrm{MnSO}_{4}\right)$ : 0.8, L-arabinosa: 20.

Antes de la realización del ensayo de optimización propiamente dicho, la actividad enzimática obtenida fue de 15,865 $\pm 3,54 \mathrm{U} \mathrm{ml}^{-1}$ empleando el medio MRS modificado oportunamente diseñado para la obtención de las primeras preparaciones enzimáticas. Así y luego, de realizado el ensayo experimental de Taguchi, se determinó el mejor nivel para cada componente, resultando en los datos observados en la Tabla 4. Asimismo, la concentración de proteínas creció de $0,58 \mathrm{mg} \mathrm{ml}^{-1}$ a $15,2 \mathrm{mg} \mathrm{ml}^{-1}$, indicando un aumento de más de 26 veces. El 
resultado máximo obtenido fue el correspondiente a la experiencia 15 del ensayo, el cual arrojó un valor de actividad enzimática de 509,360 \pm 0,242 $\mathrm{U} \mathrm{ml}^{-1}$. Esto implica un aumento de más de 36 veces en relación a la actividad enzimática obtenida en preparaciones de medios modificados no optimizadas. Las condiciones óptimas fueron las utilizadas en la elaboración posterior de los nuevos medios MRS modificados para la producción de los extractos libres de células provenientes de E. faecium DBFIQ E36.

Dado estos resultados promisorios, se encuentra efectuándose un estudio de optimización más amplio que tiene en cuenta las interacciones y los factores principales más influyentes en base a los resultados informados en este trabajo.

\section{REFERENCIAS}

BOUDEBBOUZE, S.; MAGUIN, E.; Rhimi, M. Bacterial L-Arabinose Isomerases: Industrial Application for D-Tagatose Production.Recent Pat. DNAGene Seq., v. 5, p. 194-201, 2011.

BRADFORD, M. M. A Rapid and Sensitive Method for the Quantitation of Microgram Quantities of Protein Utilizing the Principle of Protein-Dye Binding. Anal. Biochem., v. 72, p. 248-254, 1976.

DESHMUKH, S. S.;DESHPANDE, M.V.;SHANKAR, V.Medium Optimization for the Production of Glucose Isomerase from Thermophilic Streptomyces thermonitrificans. World J. Microbiol. Biotechnol., v. 10, p. 264-267, 1994.

DOBROGOSZ, W. J.;DeMOSS, R. D. Pentose Utilization by Pediococcus pentosaceus. J. Bacteriol., v. 85, p. 1356-1364, 1963.

GIVRY, S. y F. DUCHIRON, Optimization of Culture Medium and Growth Conditions for Production of L-Arabinose Isomerase and D-Xylose Isomerase by Lactobacillus bifermentans.Mikrobiologiia,v. 77, p. 281-287, 2008.

GROTHE, E., MOO-YOUNG, M.; CHISTI, Y. Fermentation Optimization for the Production of Poly ( $\beta$-hydroxybutyric acid) Microbial Thermoplastic. Enz. Microb. Technol., v. 25, p. 132-141, 1999.

HEATH, E. C.; Horecker, B. L.; Smyrniotis, P. Z.; Takagi, Y. Pentose Fermentation by Lactobacillus plantarum: II. L-arabinose Isomerase.J. Biol. Chem., v. 231, p. 10311037, 1958.

IM, J. -H.;SONG, J. -M.; KANG, J. -H.; KANG,D. -J. Optimization of Medium Components for High-Molecular-Weight Hyaluronic Acid Production by Streptococcus sp. ID9102 via a Statistical Approach.J. Ind. Microbiol. Biotechnol., v. 36, p. 1337-1344, 2009.

KULKA, R. Colorimetric Estimation of Ketopentoses and Ketohexoses. Biochem. J., p. 542$548,1956$.

MANZO, R. M.;SIMONETTA, A. C.;RUBIOLO, A. C.;MAMMARELLA, E. J. Screening and selection of wild strains for L-arabinose isomerase production. Braz. J. Chem. Eng., v. 30, p. 711-720, 2013.

MOORE, M. Drug Evaluation: Tagatose in the Treatment of Type 2 Diabetes and Obesity. Curr. Opin. Investig. Drugs, p. 924-935, 2006.

ROY,R. K. Design of Experiments using the Taguchi Approach: 16 Steps to Product and Process Improvement, Wiley-Interscience, Nueva York, 560 p, 2001.

TORRES, P. R.; MANZO, R. M.; RUBIOLO, A. C.; BATISTA-VIERA, F. D.; MAMMARELLA, E. J. Purification of an L-arabinose isomerase from Enterococcus faeciumDBFIQ E36 employing a biospecific affinity strategy. J. Mol. Catal. B: Enz.,v. 102, p. 99-105, 2014. 
YAMANAKA, K.;WOOD, W. A.L-arabinose Isomerase. MethodsEnzymol., v. 9, p. 596-602, 1966. 